\title{
Novel alleles of yeast hexokinase PII with distinct effects on catalytic activity and catabolite repression of SUC2
}

\author{
Stefan Hohmann, ${ }^{1,3}$ Joris Winderickx, ${ }^{1}$ Johannes $\mathrm{H}$. de Winde, ${ }^{1}$ \\ Dirk Valckx, ${ }^{1}$ Philip Cobbaert, ${ }^{1}$ Kattie Luyten, ${ }^{1,2}+$ \\ Catherine de Meirsman, ${ }^{1}$ José Ramos ${ }^{2}$ and Johan M. Thevelein ${ }^{1}$
}

1 Laboratorium voor Moleculaire Celbiologie, Katholieke Universiteit Leuven, Kardinaal Mercierlaan 92, B-3001 Heverlee, Flanders, Belgium

2 Departamento de Microbiologia, ETSIAM Universidad de Cordóba, E-14080 Cordóba, Spain

3 Department of Cell and Molecular

Biology/Microbiology, Göteborg University, Box 462, S-40530 Göteborg. Sweden

\author{
Author for correspondence: Johan M. Thevelein. Tel: +32 16 321507. Fax: +32 16321979. \\ e-mail: johan.thevelein@bio.kuleuven.ac.be
}

Keywords: hexokinase, catabolite repression, sugar phosphorylation, cAMP, yeast

\section{INTRODUCTION}

The addition of glucose or fructose to yeast cells growing on a non-fermentable carbon source such as ethanol causes a global switch in metabolism from gluconeo-

†Present address: Institute for Wine Biotechnology, University of Stellenbosch, Private Bag XI, 7602 Matieland, South Africa.

Abbreviation: YP, yeast extract/peptone. genesis/respiration to glycolysis/fermentation (Zimmermann \& Entian, 1997; Gancedo, 1998). Cells performing gluconeogenesis are catabolite derepressed, while fermenting cells are catabolite repressed. The transition from the derepressed to the repressed state is achieved by altering the activity and stability of enzymes and by switching the expression of a large number of genes on or off (Gancedo \& Gancedo, 1997). For instance, the half-life of mRNAs encoding enzymes involved in the utilization of sucrose and in respiration 
drops dramatically after glucose addition (Cereghino $\&$ Scheffler, 1996). Subsequently, their transcription is shut down by carbon-catabolite repression (Ronne, 1995: Gancedo, 1998). The key components of the cataboliterepression pathway are the Snf1/Cat1 protein kinase and the protein phosphatase type 1, Glc7 (Gancedo, 1998; Hardie et al., 1998). Snf1/Cat1 appears to control transcriptional regulatory proteins such as the Mig1 and Mig2 repressors (Ronne, 1995; Gancedo, 1998).

Beyond the long-known requirement of hexokinase PII activity for glucose repression (Entian, 1980), little is known about the actual sugar-sensing mechanism. Baker's yeast has three glucose- or fructose-phosphorylating enzymes: the hexokinases PI (Hxk1) and PII (Hxk2), which phosphorylate glucose and fructose (Lobo \& Maitra, 1977), and glucokinase (Glk1), which is specific for glucose (Maitra \& Lobo, 1983). Since only mutation of $H X K 2$, but not of $H X K 1$, leads to a loss in glucose repression, a unique role had been ascribed to Hxk2 in triggering glucose repression (Entian, 1980; Entian \& Fröhlich, 1984; Entian et al., 1984). Subsequent work has shown, however, that both Hxk1 and Hxk2 contribute to glucose and fructose repression (Hohmann, 1987; Rose et al., 1991; de Winde et al., 1996). Moreover, expression of HXK1 and GLK1 is glucose repressible (Sierkstra et al., 1992; Herrero et al., 1995; de Winde et al., 1996), providing a simple explanation for the predominant role of $\mathrm{Hxk} 2$ in maintaining glucose repression.

Initially, it had been proposed that $\mathrm{Hxk} 2$ has a specific role in signalling besides its catalytic function (Entian $\&$ Fröhlich, 1984). Subsequently, a good inverse correlation between hexokinase activity and the degree of catabolite repression has been reported (Ma et al., 1989; Rose et al., 1991) suggesting that the level of sugar phosphates might trigger catabolite repression. This would imply the existence of a system that senses the level of such metabolites, but despite extensive genetic analysis of catabolite repression and glycolytic regulation no such sensor has been found (Ronne, 1995; Zimmermann \& Entian, 1997; Gancedo, 1998).

Yeast tps1 mutants are deficient in growth on rapidly fermented sugars like glucose and fructose because of an unrestricted influx of sugar into glycolysis, which leads to a hyperaccumulation of sugar phosphates and depletion of ATP and phosphate (Thevelein \& Hohmann, 1995). Apparently these mutants lack a feedback control of glycolysis on hexokinase activity. The precise regulatory mechanism is not well understood (Blázquez et al., 1993; Hohmann et al., 1996; Ernandes et al., 1998). Reduction of hexokinase activity by deletion of $H X K 2$ restores growth of the tps1 $1 \Delta$ mutant on glucose, but not on fructose (Hohmann et al., 1993).

In this work we have exploited the growth defect of the tps1 $1 \Delta x k 1 \Delta$ mutant on fructose (Hohmann et al., 1993; Van Aelst et al., 1993) in a search for novel regulators of glycolysis and sugar-induced signalling. Unexpectedly, all the mutations studied define alleles of $H X K 2$ with interesting novel properties. For the first time, mutant alleles of $H X K 2$ are described in which the capacity to phosphorylate glucose or fructose is differentially affected. We also describe two mutant alleles that confer very different in vitro and in vivo sugar kinase activity while causing very similar defects in catabolite repression.

\section{METHODS}

Strains and growth conditions. The yeast strains used were all isogenic to W303-1A (Thomas \& Rothstein, 1989). The construction of the deletions of TPS1 (Hohmann et al., 1993), HXK1, HXK2 and GLK1 (Rose et al., 1991) have been described previously and the mutant strains in the W303-1A background have been listed elsewhere (de Winde et al., 1996). Strains were grown on standard yeast extract/peptone (YP) media or yeast nitrogen base/ammonium sulphate media supplemented with $2 \%$ carbon source, as indicated (Sherman et al., 1983).

Mutant isolation. For the isolation of suppressor mutations, strain YSH 311 (MATa leu2-3,112 ura3-1 trp1-1 his3-11,15

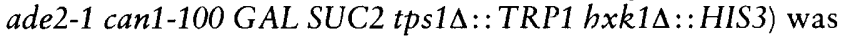
grown to saturation in YP plus $2 \%$ galactose and approximately $2 \times 10^{7}$ cells per plate were spread onto YP medium plus $2 \%$ fructose. Colonies appearing after $3-5 \mathrm{~d}$ were spread again on the same medium in order to obtain single colonies. Strains YSH 369 (MAT $\alpha$ leu2-3,112 ura3-1 trp1-1 his3-11,15

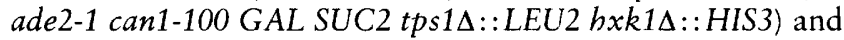
YSH 6.59.-4A (MAT leu2-3,112 ura3-1 trp1-1 his3-11,15 ade2-1 can1-100 GAL SUC2 hxk2A::LEU2) were used for genetic analysis. Crossings and tetrad analysis were done according to standard procedures (Sherman et al., 1983).

Sequence analysis. To determine mutations in the different HXK2 alleles, fragments covering the complete gene were amplified using standard PCR amplification of total genomic DNA. Sequence data were obtained by analysis of three independently cloned PCR fragments and were confirmed by direct sequence determination of the different PCR products (T7 sequenase kits, USB-Amersham).

Blotting techniques. SDS-PAGE was performed on $10 \%$ separating gels as described by Laemmli (1970). Immunoblotting was performed according to Towbin et al. (1979) using an antiserum raised against commercially available purified hexokinase (Boehringer Mannheim) as the primary antibody at a $1 / 10000$ dilution and peroxidase-labelled goat anti-rabbit IgG as the secondary antibody at a $1 / 2000$ dilution. Northern-blot analysis was performed essentially as described by Crauwels et al. (1997).

Biochemical analyses. For determination of specific hexokinase activity, cells were grown in YP medium supplemented with $4 \%$ of either glucose or fructose and harvested in the lateexponential growth phase. Crude extracts were prepared in $100 \mathrm{mM}$ potassium phosphate buffer $\mathrm{pH} 6.5$ and the activity was measured as described by Lobo \& Maitra (1977). To measure specific invertase activity, cells were grown and treated as for the hexokinase assay and the activity was determined according to Goldstein \& Lampen (1975). For cAMP determination, cells were grown on YP medium supplemented with $3 \%$ glycerol and $0 \cdot 1 \%$ galactose until lateexponential phase. Incubation with glucose and fructose (100 $\mathrm{mM}$ each) and quantification of cAMP followed our established protocol (Thevelein et al., 1987). The same culturing regime was used for cells in which the levels of glycolytic metabolites were determined according to 
Novel yeast hexokinase mutations

de Koning \& van Dam (1992). For the determination of ethanol production, cells were grown in $100 \mathrm{ml}$ medium in $250 \mathrm{ml}$ Erlenmeyer flasks in a shaker at 300 r.p.m. Samples were taken at different time points and the ethanol in the medium supernatant was determined using the test combination ethanol kit from Boehringer Mannheim.

Sugar transport. The amount of ${ }^{14} \mathrm{C}$-labelled glucose or fructose taken up within $5 \mathrm{~s}$ was measured in exponentially growing cells as described previously (Luyten et al., 1993).

Reproducibility of data. All experiments were performed at least in triplicate from independent cultures. These different experiments gave consistent trends, i.e. the differences between strains were highly reproducible. The absolute values for enzyme activities, metabolite concentration and relative mRNA levels varied between different independent experiments by not more than $30 \%$. The results from representative experiments are shown.

\section{RESULTS}

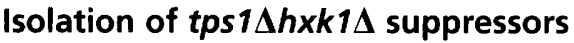

We have shown previously that deletion of the HXK2 gene suppresses the growth defect of a tps $1 \Delta$ mutant on glucose but not on fructose medium (Hohmann et al., 1993). We have also observed that most of the spontaneous suppressor mutations of a $t p s 1 \Delta$ mutant isolated on glucose medium are either allelic to $H X K 2$ or cause a petite phenotype (unpublished observations and Blázquez \& Gancedo, 1995). To easily identify petite mutants, we made use of the fact that they do not develop the typical red colour in an ade 2 background like W303-1A. For the isolation of suppressors we used a tps1 $h x k 1 \Delta$ double mutant, which has a growth defect on glucose and fructose that is indistinguishable from that of the tps1 $\Delta$ single mutant (Hohmann et al.,

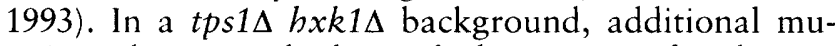
tations that strongly diminish the activity of $\mathrm{Hxk} 2 \mathrm{can}$ be recognized easily since the resulting strain should not grow on fructose medium when respiration is blocked by antimycin A (Lobo \& Maitra, 1977).

We isolated 128 fructose-positive red mutants from the W303-1A derivative YSH 311 (MATa tps1A::TRP1 hxk1د::HIS3). All but 12 of these mutants failed to grow on fructose medium containing the respiration inhibitor antimycin A and were thus likely to have strongly reduced $\mathrm{Hxk} 2$ activity. The remaining 12 mutations segregated $2: 2$ for suppression of the growth defect of the tps1 $1 \Delta:: L E U 2 h \times k 1 \Delta:: H I S 3$ strain, showing that this phenotype was due to a single nuclear mutation in each case. When crossed with an isogenic $h x k 2 \Delta:: L E U 2$ strain, the new mutations cosegregated with the $h x k 2 \Delta$ allele in all 8-12 complete tetrads tested for each mutant, suggesting allelism with $H X K 2$. Since all putative tps $1 \Delta b x k 1 \Delta b x k 2$ spores could grow well on fructose plus antimycin, it appeared that the mutants still retained sufficient hexokinase activity to permit fructose fermentation. Consistent with this, the new $h x k 2$ mutations were unable to suppress the growth defect of the tps $1 \Delta$ mutant on fructose in the presence of a wild-type $H X K 1$ gene, probably because hexokinase activity was too high in such strains.
Table 1. Mutations found in the $H X K 2$ alleles isolated as suppressors of the growth defect of a $\operatorname{tps} 1 \Delta h \times k 1 \Delta$ strain on fructose

\begin{tabular}{|ll|}
\hline Allele & Mutation \\
\hline$b x k 2-36$ & Pro-160 $\rightarrow$ Ala \\
$b x k 2-37$ & Ala $-132 \rightarrow$ Pro \\
$b x k 2-39$ & Asp-343 $\rightarrow$ Glu \\
$h x k 2-53$ & Asp-179 $\rightarrow$ Gly \\
$h x k 2-97$ & Tyr-346 $\rightarrow$ Asp \\
$h x k 2-129$ & Glu-456 $\rightarrow$ Gly \\
\hline
\end{tabular}

Suppression of the $t p s 1 \Delta h x k 1 \Delta$ growth defect by the new $h \times k 2$ alleles is most probably due to diminished hexokinase activity, and not to some other property of the mutant Hxk2. When some of the new alleles (nos 39, 53 and 97, see below) were cloned and expressed from a multi-copy plasmid, they conferred hexokinase activity 5-10 times higher than that of an untransformed wildtype and consequently failed to suppress a $t p s 1 \Delta$ mutant (results not shown). Also the semi-dominant character exibited by all the novel $h x k 2$ mutations with respect to suppression of a tps1s mutation (data not shown), which is also apparent for a deletion of $H X K 2$, is probably due to reduction of the specific hexokinase activity in the heterozygous diploids. All the heterozygous diploids tested, including the $H X K 2 / h \times k 2 \Delta$ strain, had a specific hexokinase activity between 60 and $70 \%$ of the wild-type.

\section{Analysis of novel $H X K 2$ alleles}

All further analyses were done in strains that carried a wild-type TPS1 gene. The 12 mutants fell into six distinct groups with respect to the specific hexokinase activity and their ability to mediate catabolite repression of invertase activity (data not shown). One typical mutant from each group was analysed further. The $H X K 2$ gene from each of the mutant strains carried one nucleotide change leading to a substitution of one a mino acid (Table 1). The cloned alleles 39, 53 and 97 were retransformed into yeast on multi-copy plasmids. None of these alleles was able to confer the same high hexokinase activity as found in a strain transformed with the same plasmid carrying the wild-type $H X K 2$ gene (data not shown).

Table 2 shows the specific hexokinase activity conferred by the six selected $H X K 2$ alleles and that of control strains. Deletion of HXK1 and/or GLK1 caused only a marginal reduction in the specific hexokinase activity, consistent with the predominant expression of HXK2 during growth on glucose. On fructose medium, HXK1 is also expressed to a moderate extent (de Winde et al., 1996). All six HXK2 mutant alleles caused diminished specific hexokinase activity, although to a very different extent. Interestingly, fructose phosphorylation was more severely affected than glucose phosphorylation in

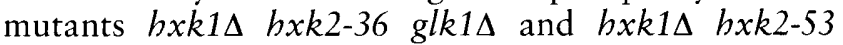
glk1 1 . Note that mutant $h x k 1 \Delta h x k 2-39$ glk1 $\Delta$ conferred 
Table 2. Hexokinase and invertase activities in strains containing specific hexokinase mutations

\begin{tabular}{|c|c|c|c|c|c|c|c|c|}
\hline \multirow{2}{*}{$\begin{array}{l}\text { Strain (relevant } \\
\text { genotype) }\end{array}$} & \multicolumn{4}{|c|}{ Growth on glucose } & \multicolumn{4}{|c|}{ Growth on fructose } \\
\hline & $\begin{array}{c}\text { Hxk } \\
\text { activity } \\
(\text { fructose })^{*}\end{array}$ & $\begin{array}{c}\text { Hxk } \\
\text { activity } \\
\text { (glucose) }^{*}\end{array}$ & F/G ratio $\dagger$ & $\begin{array}{l}\text { Invertase } \\
\text { activity }^{*}\end{array}$ & $\begin{array}{c}\text { Hxk } \\
\text { activity } \\
(\text { fructose })^{*}\end{array}$ & $\begin{array}{c}\text { Hxk } \\
\text { activity } \\
\text { (glucose) }\end{array}$ & $\mathrm{F} / \mathrm{G}$ ratio $\dagger$ & $\begin{array}{r}\text { Invertase } \\
\text { activity }^{*}\end{array}$ \\
\hline$H X K 1 H X K 2$ GLK1 & 660 & 660 & $1 \cdot 00$ & 70 & 690 & 630 & $1 \cdot 10$ & 80 \\
\hline$h x k 1 \Delta H X K 2$ GLK1 & 630 & 660 & $1 \cdot 05$ & 70 & 620 & 640 & 0.97 & 80 \\
\hline$H X K 1$ hxk2 $2 \Delta L K 1$ & 490 & 210 & $2 \cdot 33$ & 1200 & 310 & 120 & $2 \cdot 58$ & 300 \\
\hline$h x k 1 \Delta b x k 2 \Delta$ GLK1 & 10 & 150 & $0 \cdot 07$ & 7000 & $<5$ & 80 & - & 1100 \\
\hline HXK1 HXK2 glk1ه & 680 & 670 & $1 \cdot 01$ & 80 & 710 & 630 & $1 \cdot 13$ & 80 \\
\hline 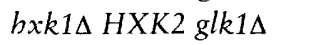 & 600 & 600 & $1 \cdot 00$ & 80 & 600 & 590 & 1.02 & 80 \\
\hline 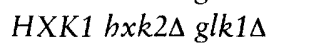 & 520 & 160 & $3 \cdot 25$ & 1250 & 240 & 50 & $4 \cdot 8$ & 200 \\
\hline$b x k 1 \Delta b x k 2 \Delta$ glk1s & $<5$ & $<5$ & - & 400 & $<5$ & $<5$ & - & 1400 \\
\hline$h x k 1 \Delta$ hxk2-36 glk1s & 50 & 270 & $0 \cdot 19$ & 90 & 50 & 280 & $0 \cdot 18$ & 110 \\
\hline 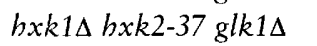 & 20 & 20 & 1.00 & 170 & 5 & 5 & $1 \cdot 00$ & 150 \\
\hline 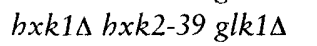 & 110 & 100 & $1 \cdot 10$ & 1300 & 100 & 70 & $1 \cdot 43$ & 480 \\
\hline 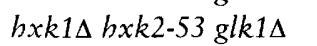 & 150 & 250 & $0 \cdot 60$ & 100 & 170 & 280 & 0.61 & 100 \\
\hline 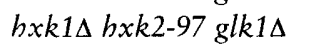 & 170 & 170 & 1.00 & 130 & 190 & 180 & $1 \cdot 05$ & 110 \\
\hline 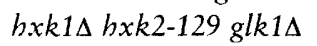 & 410 & 410 & 1.00 & 1500 & 340 & 300 & $1 \cdot 13$ & 680 \\
\hline
\end{tabular}

$* \mu \mathrm{mol} \min ^{-1}$ (mg protein $)^{-1}$. $†$ Activity with fructose divided by activity with glucose.

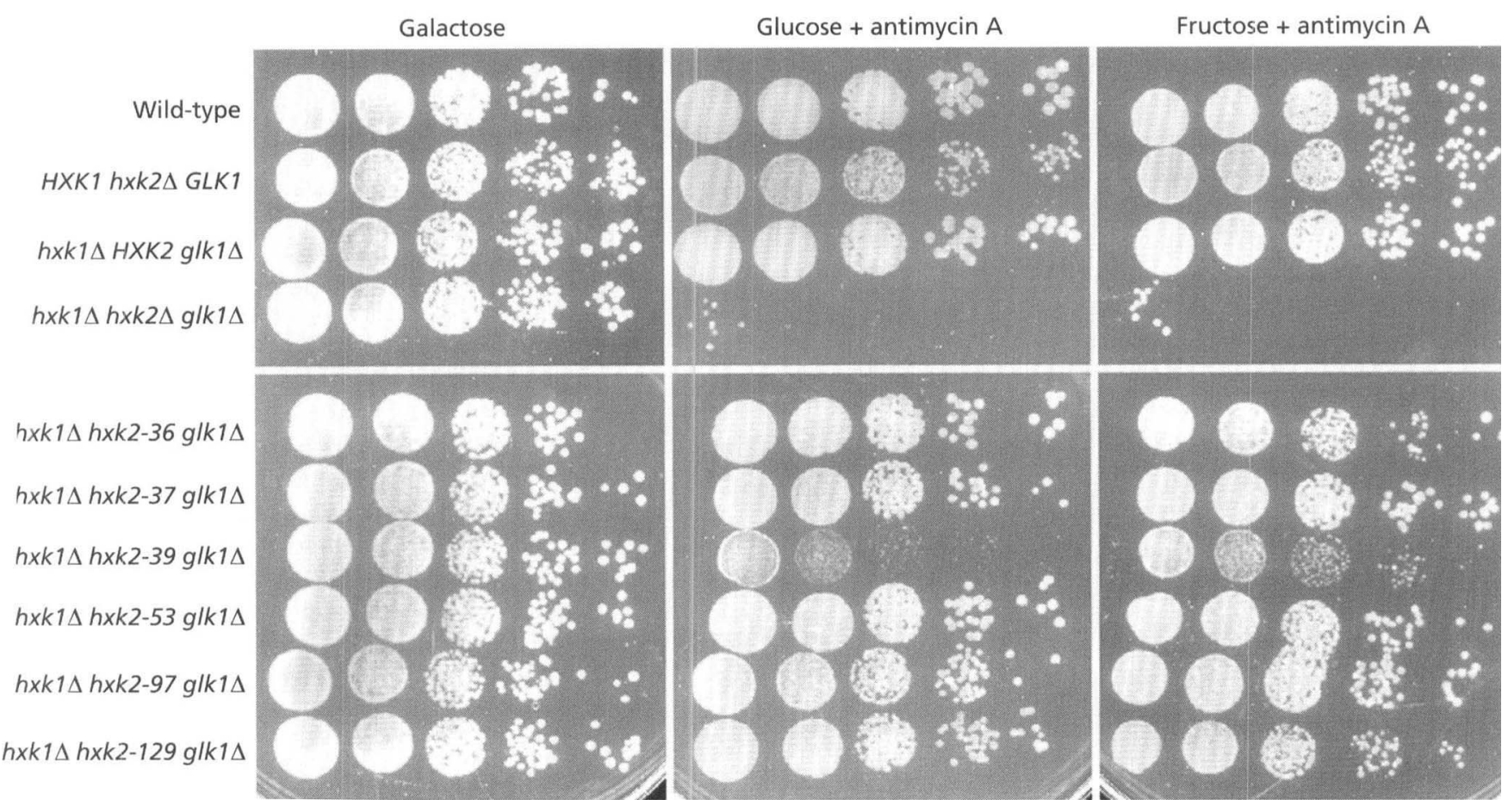

Fig. 1. Growth properties of strains carrying different hexokinase mutations. Strains were pregrown in complete (YP) medium with $2 \%$ galactose; $10 \mu \mathrm{l}$ serial $1: 10$ dilutions were spotted onto YP medium containing $2 \%$ galactose, $2 \%$ glucose and $2 \%$ fructose. The plates with glucose and fructose contained 1 p.p.m. of the respiration inhibitor antimycin $\mathrm{A}$.

less than $20 \%$ of the hexokinase activity of the strain expressing wild-type $H X K 2$ while mutant $h x k 1 \Delta b x k 2$ 129 glk1 $1 \Delta$ showed the highest residual hexokinase activity (approx. $70 \%$ ).
The growth properties of each mutant were tested by spotting serial dilutions of galactose-grown cells on medium containing glucose or fructose as the carbon source (Fig. 1). All strains grew normally on medium 
Table 3. Enzymic properties of the mutant Hxk2 proteins

\begin{tabular}{|c|c|c|c|c|c|c|c|c|}
\hline \multirow[t]{2}{*}{ Strain (relevant genotype) } & \multicolumn{2}{|c|}{ Glucose } & \multicolumn{2}{|c|}{ Fructose } & \multicolumn{2}{|c|}{ ATP } & \multicolumn{2}{|c|}{$K_{\mathrm{m}}$ sugar $/ K_{\mathrm{m}}$ ATP } \\
\hline & $K_{\mathrm{m}}^{*}$ & $V_{\max } \dagger$ & $\boldsymbol{K}_{\mathrm{m}}^{*}$ & $V_{\max } \dagger$ & $K_{\mathrm{m}}^{*}$ & $V_{\max } \dagger$ & Glc/ATP & Fru/ATP \\
\hline 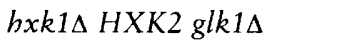 & $0 \cdot 16$ & 215 & $0 \cdot 61$ & 250 & $0 \cdot 10$ & 220 & $1 \cdot 6$ & $6 \cdot 1$ \\
\hline 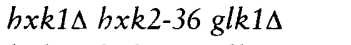 & $0 \cdot 23$ & 145 & $5 \cdot 10$ & 235 & $2 \cdot 11$ & 255 & $0 \cdot 1$ & $2 \cdot 4$ \\
\hline$b x k 1 \Delta h x k 2-37$ glk1 & $0 \cdot 31$ & 110 & $2 \cdot 46$ & 170 & $0 \cdot 37$ & 190 & $0 \cdot 8$ & $6 \cdot 6$ \\
\hline bxk1 $1 \Delta x k 2-39$ glk $1 \Delta$ & $0 \cdot 31$ & 175 & $1 \cdot 00$ & 165 & $1 \cdot 10$ & 240 & $0 \cdot 3$ & $0 \cdot 9$ \\
\hline 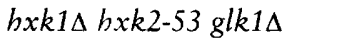 & $0 \cdot 47$ & 255 & $2 \cdot 01$ & 410 & 0.43 & 370 & $1 \cdot 1$ & $4 \cdot 7$ \\
\hline 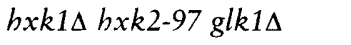 & $0 \cdot 53$ & 175 & $2 \cdot 20$ & 245 & $0 \cdot 46$ & 190 & $1 \cdot 2$ & $4 \cdot 8$ \\
\hline$h x k 1 \Delta h x k 2-129$ glk1 $1 \Delta$ & $0 \cdot 54$ & 175 & $2 \cdot 46$ & 95 & $0 \cdot 01$ & 130 & $54 \cdot 0$ & $246 \cdot 0$ \\
\hline
\end{tabular}

* mM. $† \mathrm{mmol} \mathrm{min}^{-1}$.

without the respiration blocker antimycin A (not shown). In the presence of antimycin A, only strain $h x k 1 \Delta h x k 2-39$ glk1 $\Delta$ had a clear growth problem. The in vivo hexokinase activity of mutant $h x k 1 \Delta b x k 2-36$ $g l k 1 \Delta$ with fructose and $h x k 1 \Delta b x k 2-37 g l k 1 \Delta$ with both substrates is probably higher than that monitored in vitro, since these strains grew normally (Fig. 1).

Neither the hexokinase protein level as measured by Western blot analysis nor the $H X K 2 \mathrm{mRNA}$ level as monitored by Northern blot analysis was altered in the $h x k 2$ mutants as compared to the wild-type HXK2 allele (data not shown).

\section{Properties of the $h x k 2$ products}

The kinetic properties of the mutant $\mathrm{Hxk} 2 \mathrm{~s}$ were determined in crude extracts prepared from strains in a

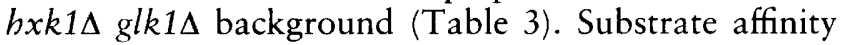
was affected to a different extent in the six mutants tested: Hxk2-36 and Hxk2-37 showed a reduced affinity for fructose and for ATP, Hxk2-39 had reduced affinity for ATP, and Hxk2-53 and Hxk2-97 had slightly reduced affinity for all three substrates. Hxk2-129 had a somewhat reduced affinity for the sugars but, remarkably, a tenfold higher affinity than the wild-type enzyme for ATP. The relative affinities for glucose/ATP of Hxk2-36 and for both glucose/ATP and fructose/ATP of Hxk239 and Hxk2-129 were strongly altered. Only mutants Hxk2-37 and Hxk2-129 showed a markedly reduced $V_{\max }$ with glucose or fructose as substrate, respectively, while Hxk2-53 had an increased $V_{\text {max }}$. Competitive inhibition of the mutant hexokinases by trehalose 6phosphate (Blázquez et al., 1993) was not significantly altered compared to that of wild-type Hxk2 (data not shown).

\section{Glycolytic metabolism in the novel $h x k 2$ mutants}

To estimate the in vivo activity conferred by the mutant $h x k 2$ alleles, we determined the rate of accumulation of glucose 6-phosphate and fructose 6-phosphate after the addition of glucose or fructose. Because of the rapid initial accumulation of sugar phosphates in tps1 $\Delta$ mutants after sugar addition, we presume that sugar uptake is not limiting at this stage (Van Aelst et al., 1993). The strains were pregrown in glycerol medium, which in wild-type cells allows expression of all three sugar-kinase genes (HXK1, HXK2 and GLK1). This explains why, after glucose addition, sugar phosphate levels were lower in the $h x k 1 \Delta$ glk1 $1 \Delta$ mutant than in the wild-type (Fig. 2).

Sugar phosphate accumulation was affected in all mutants to a different extent. In mutant $h x k 1 \Delta b x k 2-36$ glk1 $1 \Delta$, the high glucose 6-phosphate peak after addition of glucose and the relatively low fructose 6-phosphate accumulation after fructose addition were consistent with the specific hexokinase activity determined in vitro. In contrast, mutant $h x k 1 \Delta b x k 2-37$ glk1 $1 \Delta$ showed an intermediate glucose 6-phosphate and a high fructose 6phosphate peak, indicating that the in vivo activity of Hxk2-37 is much higher than that measured in vitro, as already inferred from the growth properties. The $h x k 1 \Delta$ bxk2-39 glk1 $1 \Delta$ strain showed the most severe reduction in sugar phosphate accumulation both after glucose and fructose addition of all mutants studied.

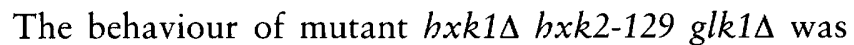
very different from that of all the other strains. The sugar phosphate levels $30 \mathrm{~s}$ after sugar addition were high, in agreement with the relatively high in vitro hexokinase activity. However, after this first time point, sugar phosphate levels did not drop in this mutant but rather were even higher than that of the wild-type after 300 s. Also, mutant $h x k 1 \Delta$ hxk2-53 glk1s showed aberrant kinetics of sugar phosphate accumulation. With both sugar substrates the product level increased slowly, reaching the highest value only at the second or third time point.

High-affinity sugar uptake by derepressed cells within the first $5 \mathrm{~s}$ after the addition of radioactive glucose or fructose is dependent on sugar kinases (Bisson \& Fraenkel, 1983; Smits et al., 1996). All the mutants showed high-affinity glucose or fructose uptake under these conditions, consistent with the presence of active hexokinase. However, high-affinity glucose and fructose 

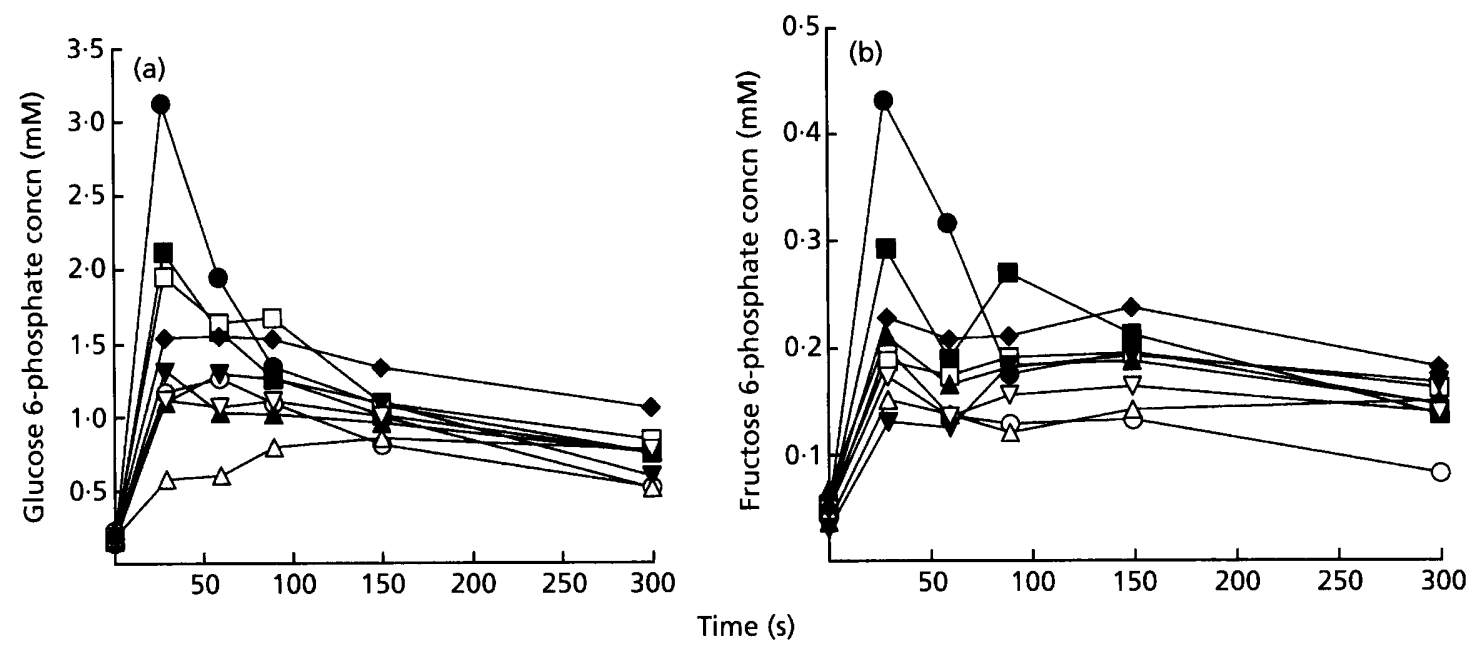

Fig. 2. Accumulation of the product of the hexokinase reaction after addition of $100 \mathrm{mM}$ substrate to glycerol-grown cells in vivo. (a) Glucose 6-phosphate after glucose addition. (b) Fructose 6-phosphate after fructose addition.

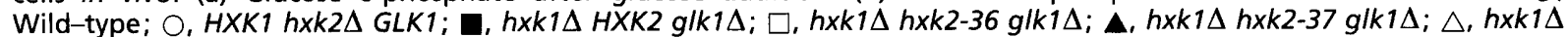

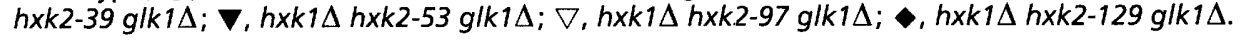
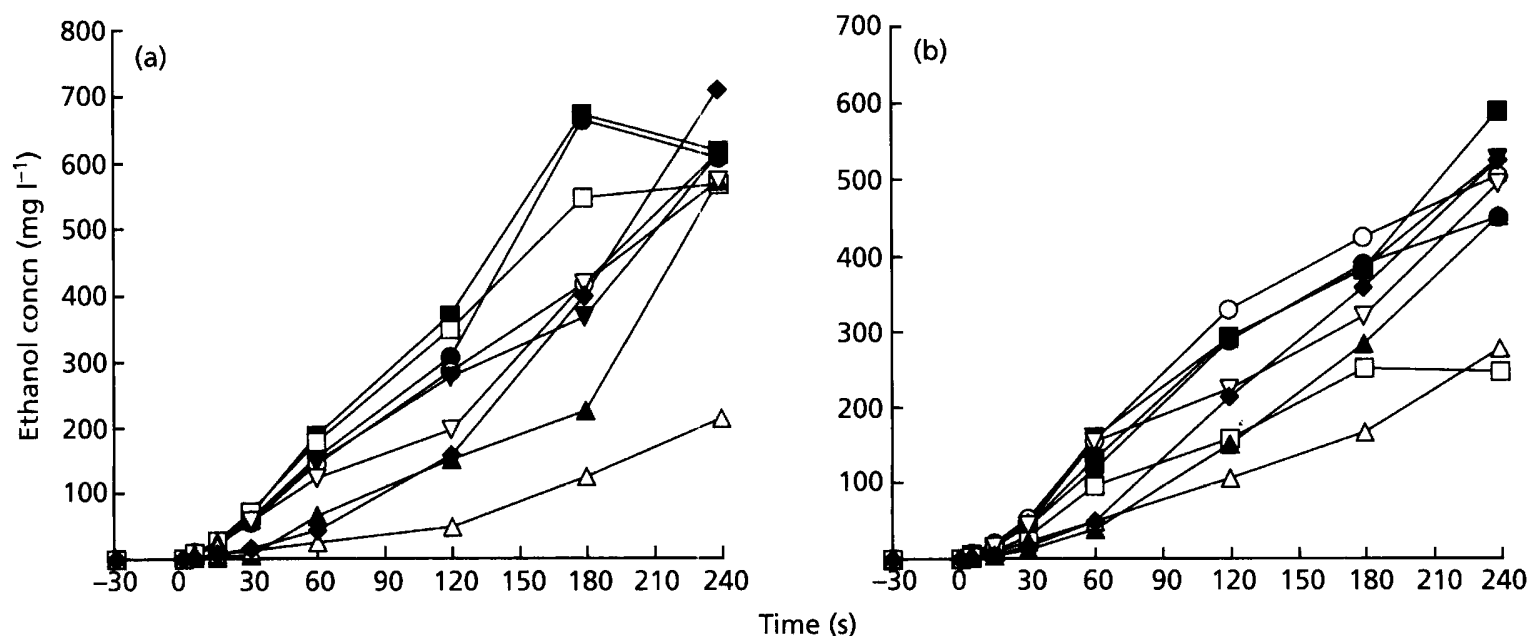

Fig. 3. Ethanol production after the addition of $100 \mathrm{mM}$ glucose (a) or fructose (b) to glycerol-grown cells. Wild-type;

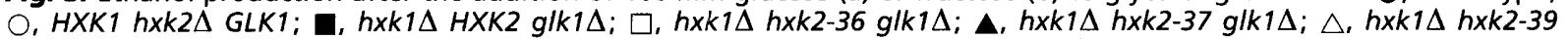

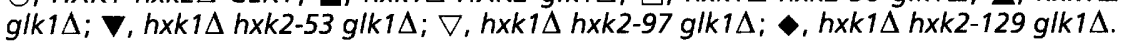

uptake were reduced in mutant $h x k 1 \Delta b x k 2-39 \mathrm{glk} 1 \Delta$ as well as - to a lesser extent - in mutants $h x k 1 \Delta h x k 2-53$

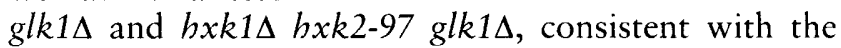
lower sugar phosphate accumulation of these three mutants (data not shown).

To estimate how the novel HXK2 mutations affect glycolytic flux, we measured ethanol production in the six selected mutants during growth on glucose (Fig. 3). The wild-type, the $h \times k 2 \Delta$ mutant and the $h x k 1 \Delta$ glk1 $\Delta$ strain showed the same ethanol production rate. Also, the profile of the mutants $h x k 1 \Delta b x k 2-53$ glk1 $1 \Delta$ and $h x k 1 \Delta h x k 2-97 \mathrm{glk1} \Delta$ was almost indistinguishable from that of the wild-type on both sugars. The ethanolproduction rate of mutant $h x k 1 \Delta h \times k 2-36$ glk1 $\Delta$ was only diminished in fructose medium, consistent with its hexokinase activity being more affected with fructose as

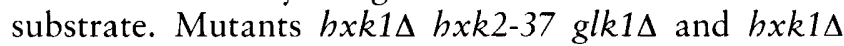
$h x k 2-129$ glk1 $\Delta$ showed a longer lag phase before their ethanol-production rate approached that of the wildtype. The ethanol production rate of mutant $h \times k 1 \Delta$ hxk2-39 glk1 $1 \Delta$ was strongly reduced on both glucose and fructose, consistent with the low sugar phosphate accumulation.

\section{Establishment of catabolite repression of the SUC2 gene for invertase}

The SUC2 gene for invertase is usually used as a marker for catabolite repression, since its expression only 
Novel yeast hexokinase mutations

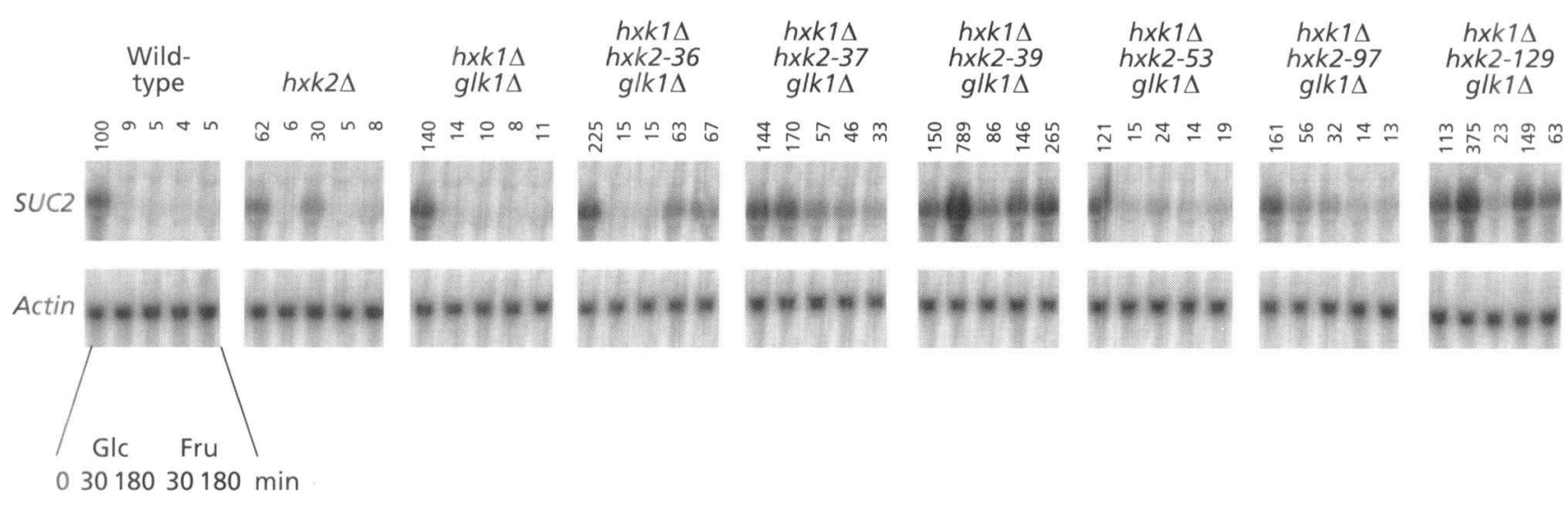

Fig. 4. SUC2 mRNA levels after the addition of $100 \mathrm{mM}$ glucose or fructose to raffinose-grown cells. Levels (numbers above lanes) were quantified with respect to actin mRNA, which is constant under these growth conditions. The wild-type mRNA level at time zero was set to 100.

responds to the glucose and fructose levels in the growth medium. The specific activity of invertase shows strong derepression on glucose medium in the absence of $H X K 2$ and even more in the absence of $H X K 2$ and $H X K 1$ (Table 2). On fructose medium, deletion of HXK2 causes only slight derepression, as previously observed (Hohmann, 1987; de Winde et al., 1996). The mutant alleles $h x k 2-36, h x k 2-53$ and $h x k 2-97$ mediated virtually normal repression of invertase activity. Mutants $h x k 1 \Delta$

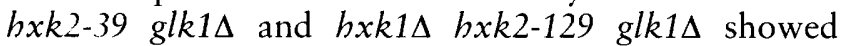
derepression of the invertase activity in glucose medium very much like the $h x k 2 \Delta$ mutant. This is particularly remarkable for allele $h x k 2-129$, since it showed the highest specific-hexokinase activity of all six mutants investigated. In addition, mutants $h \times k 1 \Delta h \times k 2-39 \mathrm{glk} 1 \Delta$ and $h \times k 1 \Delta b \times k 2-129 \mathrm{glk} 1 \Delta$ had higher invertase activity than the $h x k 2 \Delta$ mutant in fructose medium.

We monitored by Northern-blot analysis short- and long term catabolite repression of SUC2 mRNA by taking samples 30 and $180 \mathrm{~min}$ after the addition of glucose or fructose to raffinose-grown cells from the six $h x k 2$ mutants in a $h x k 1 \Delta$ glk1 $1 \Delta$ background and the

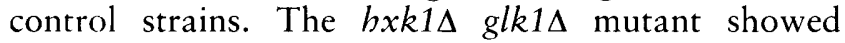
catabolite repression exactly like the wild-type (Fig. 4). The $h x k 2 \Delta$ mutant showed short-term glucose repression but long-term repression in glucose medium was severely affected. Fructose repression in the $b x k 2 \Delta$ mutant was comparable to that of the wild-type, as reported previously (de Winde et al., 1996). Each of the six $h \times k 1 \Delta$ glk $1 \Delta$ bxk2 mutant strains exhibited a unique pattern of SUC2 expression with respect to short- and long-term catabolite repression. Mutant $h x k 1 \Delta h x k 2-53$ $g l k 1 \Delta$ behaved almost like the wild-type. Mutant $h x k 1 \Delta$ hxk2-36 glk1 1 , which had specifically low fructosephosphorylating activity in vitro, did not completely repress the SUC2 mRNA level after fructose addition but showed normal glucose-repression. Thus, this is the first $h \times k 2$ mutant specifically affected in fructose repression. Mutant $h x k 1 \Delta b x k 2-97 \mathrm{glk1}$, on the other hand, showed only partial short- and long-term glucose repression but normal fructose repression. Mutant $h \times k 1 \Delta h \times k 2-37 g l k 1 \Delta$ behaved in a similar way, although fructose repression was only partially affected, despite very low in vivo fructose kinase activity.

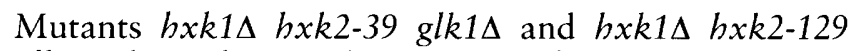
glk1 $1 \Delta$ showed a similar pattern of SUC2 expression, despite a very different hexokinase activity and sugar catabolism. Both mutants showed respectively a fiveand threefold enhanced instead of decreased SUC2 mRNA level $30 \mathrm{~min}$ after glucose addition. Long-term glucose repression, however, was almost normal in mutant $h x k 1 \Delta h x k 2-129 \mathrm{glk} 1 \Delta$ and a twofold repression could be seen also in mutant $h x k 1 \Delta$ hxk2-39 glk1 . Neither mutant showed short-term fructose repression. Mutant $h x k 1 \Delta h x k 2-39$ glk1 $\operatorname{did}$ not show long-term fructose repression, while there was a minor, twofold fructose repression of the SUC2 mRNA level in mutant

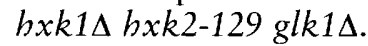

With respect to long- and short-term repression, the $h x k 2 \Delta$ and the $h x k 1 \Delta b x k 2-129$ glk1 $\Delta$ strains represent two extremes: the $h x k 2 \Delta$ mutant has normal short-term disappearance of SUC2 mRNA but lacks long-term repression. The $h x k 1 \Delta b x k 2-129$ glk1 $1 \Delta$ strain lacks the short-term effect - which is rather converted into induction - but has normal long-term glucose repression.

\section{CAMP signalling in the $h \times k 2$ mutants}

The addition of glucose or fructose to derepressed cells triggers a rapid, transient increase in the intracellular cAMP level (Thevelein \& Beullens, 1985). This effect has been shown to be dependent on sugar phosphorylation (Beullens et al., 1988). We monitored the intracellular level of cAMP in mutants expressing $H X K 2$ or the new $h \times k 2$ mutant alleles as their only source of hexokinase activity after the addition of glucose or fructose (Fig. 5). All mutants were affected in the magnitude of the glucose-induced cAMP signal. Mutant $h x k 1 \Delta h \times k 2-39$ glk1 $\Delta$ hardly showed any signal at all. In 

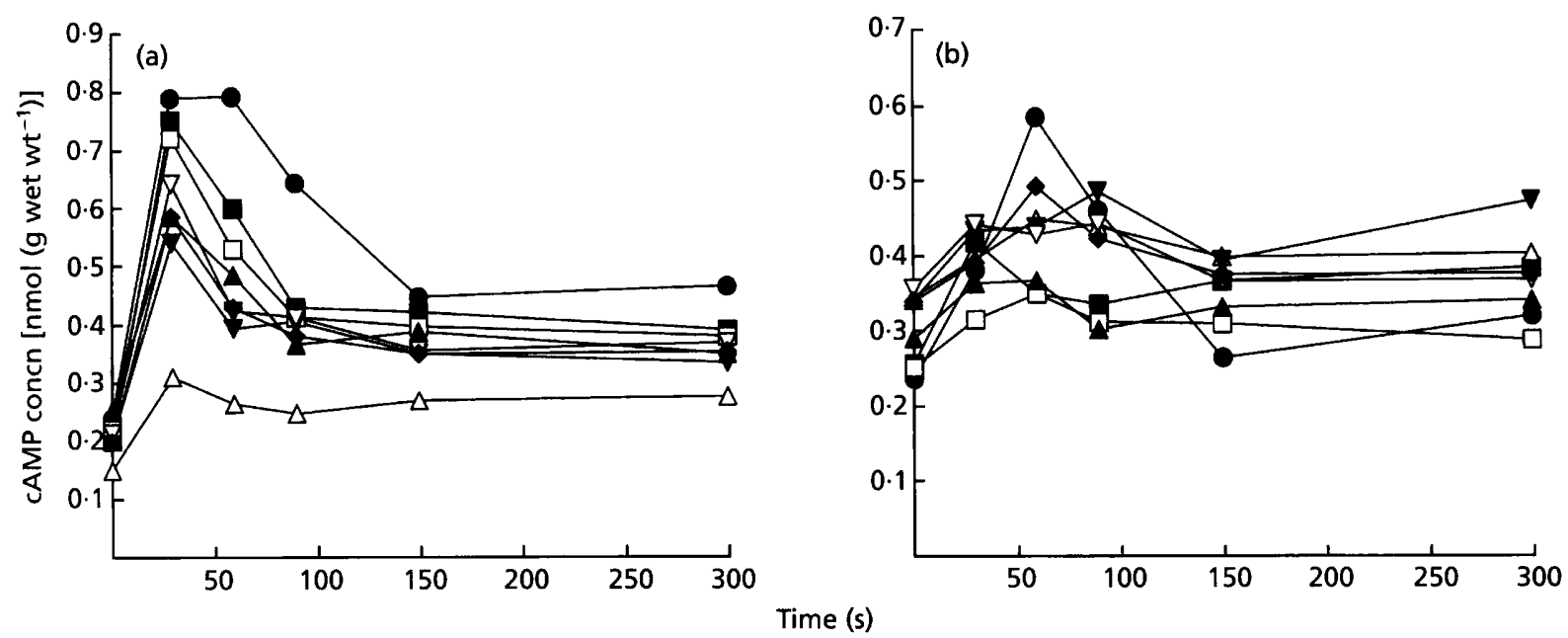

Fig. 5. CAMP content after the addition of $100 \mathrm{mM}$ glucose (a) or $100 \mathrm{mM}$ fructose (b) to glycerol-grown cells.

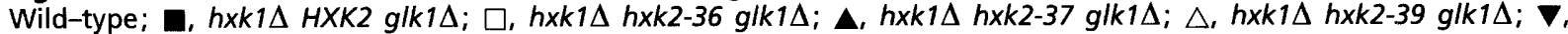
$h \times k 1 \Delta h \times k 2-53 g l k 1 \Delta ; \nabla, h \times k 1 \Delta h \times k 2-97 g l k 1 \Delta ; \bullet, h \times k 1 \Delta h \times k 2-129 g l k 1 \Delta$.

none of the other mutants was the highest value less than two-thirds of that of the control strain expressing only wild-type HXK2. The pattern observed for the mutants is very similar to the glucose 6-phosphate peak (Figs 2a and 5 a). Fructose does not induce an equally clear cAMP spike like glucose. The new $h x k 2$ mutants showed, to different degrees, a broader signal with fructose than the wild-type strain.

\section{DISCUSSION}

By screening for suppression of the growth defect on

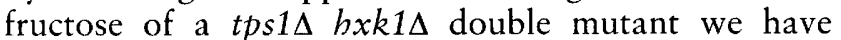
isolated novel alleles of the $H X K 2$ gene, encoding the major hexokinase isoform $\mathrm{Hxk} 2$. In fact, all respirationproficient suppressor mutants analysed were $H X K 2$ mutations. This is remarkable because the screen was initially designed to prevent the selection of $b x k 2$ mutations, which are predominant when screening for tps1 suppressors on glucose medium (our unpublished data and Blázquez \& Gancedo, 1995). We had chosen to screen for suppressors in a $t p s 1 \Delta b x k 1 \Delta$ mutant on fructose since a $h x k 1 b x k 2$ double-deletion mutant can not grow on fructose. However, there was still a strong bias towards the selection of $b x k 2$ mutants, which displayed residual catalytic activity and interesting properties. These novel $h x k 2$ mutants could be used as a tool in structure-function analysis of $\mathrm{Hxk} 2$ in the future.

The detailed analysis of the mutants identified in this work leads to five major conclusions:

1. Different mutations in $\mathrm{Hxk} 2$ can affect the catalytic activity with the two sugar substrates glucose and fructose to different extents, suggesting that the structural requirements for binding of glucose and fructose can be separated.

2. We have confirmed that the establishment of catabolite repression is at least a two-step process, with a short-term disappearance of the repressible mRNA and a long-term sustained effect. These two phases can be dissected using different alleles of $H X K 2$.

3. We have isolated for the first time a HXK2 mutation that has lost its ability to mediate fructose repression while glucose repression is still normal.

4. Not all $H X K 2$ mutants conform to the previously established inverse correlation between the catalytic activity of $\mathrm{Hxk} 2$ and its ability to mediate catabolite repression. This suggests that the role of hexokinases in catabolite repression may be more complex than simply catalytic.

5. There is a good correlation between sugar phosphate and cAMP accumulation after sugar addition to derepressed yeast cells. There is, however, no correlation between CAMP accumulation and the ability of the strain to trigger short- or long-term catabolite repression, arguing against a role for cAMP in either process.

\section{Implications for Hxk2 structure and function}

We attempted to correlate the nature and position of the affected amino acids in the published three-dimensional structure of yeast hexokinase (Anderson et al., 1978; Bennett \& Steitz, 1980) and the highly similar mammalian glucokinase (Gidh-Jain et al., 1993) with the effects on substrate recognition and catalytic activity.

The bxk2-36 mutation has remarkable consequences since it converts the enzyme from a bifunctional glucose/fructose kinase almost to a glucokinase. The mutation changes Pro- 160 to Ala. Pro- 160 is the last of a block of highly conserved amino acids in the hexokinase gene family. This ${ }^{152}$ PLGFTFSFP ${ }^{160}$ motif has previously been proposed to function in sugar binding (Schirch \& Wilson, 1987). Site-directed mutagenesis and computer- 
assisted modelling of human $\beta$-cell glucokinase (GidhJain et al., 1993) and of yeast Hxk2 have identified the neighbouring Ser-158 as an important determinant of sugar-binding affinity, sugar/ATP-binding co-operativity and of the phosphoryl transfer from ATP to the sugar (Xu et al., 1995). Indeed, Ser-158 has recently been mapped as the site of the hexokinase autophosphorylation when the enzyme is inactivated upon binding of D-xylose (Heidrich et al., 1997), stressing the central role of this amino acid in the phosphoryl transfer reaction. Mutation of Pro-160 to Ala may distort the Ser-158 region and hence affect ATP-binding and $V_{\max }$, without significant impact on glucose binding affinity. In contrast, fructose-binding affinity is severely affected in Hxk2-36, with no apparent effect on $V_{\text {max }}$. Mutation of Ser-158 itself likewise causes differential effects on binding affinity, $V_{\max }$ and sugar-induced repression kinetics for glucose and fructose (J. H. de Winde and others, unpublished). This discrepancy is indicative of different substrate recognition requirements of $\mathrm{Hxk} 2$ for glucose and for fructose. The difference appears to be reflected in the extent of catabolite repression mediated through Hxk2-36; repression is normal with glucose, but partially deficient with fructose (Fig. 4).

In Hxk2-37, Ala-132 is changed into a Pro. This mutation is likely to distort a structurally conserved $\alpha$ helix within the small lobe of hxk, affecting both sugarand ATP-binding affinity and hence, $V_{\max }$.

Mutations in Hxk2-39 (Asp-343 to Glu) and Hxk2-97 (Tyr-346 to Asp) are in neighbouring amino acids within the heart of the large lobe, away from the sugar-binding cleft. These mutations have profoundly different effects on enzyme activity, substrate recognition and sugarinduced signalling.

The $h x k 2-53$ mutation changes Asp-179 into Gly. Asp179 immediately follows the ${ }^{174}$ WTKGF $^{178}$ motif, which is highly conserved in the hexokinase family, located near the top of the small lobe. The mutation decreases all substrate affinities to a comparable extent but increases $V_{\max }$ for all substrates. Apparently, substrate recognition is not affected since sugar-induced signalling is comparable to wild-type.

Finally, the mutation in $b x k 2-129$ alters Glu-457 into a Gly. Glu-457 is part of a highly conserved motif ${ }^{457}$ EDGSGAGAAV $^{466}$ at the C-terminal end of all known members of the hexokinase family. This motif comprises a structurally conserved and important connecting helix of the ATPase domain of diverse ATP-binding proteins (Bork et al., 1992). Insertion of a glycine at the beginning of the connecting helix may tilt this helix, causing the two ATP-binding lobes to come into closer contact (Bork et al., 1992). In line with this, mutation of Glu-456 into Gly causes a 10-fold decrease in the $K_{\mathrm{m}}$ for ATP. Apparently, this structural distortion and increased ATP-binding affinity severely affect catalytic activity and substrate recognition, exemplified by severe decreases of $V_{\max }$ and abnormal sugar-induced signalling. Interestingly, the effects on sugar-induced signalling are comparable to those of Hxk2-39, which has a 10 -fold increased $K_{\mathrm{m}}$ for ATP. This again separates kinetic properties of hexokinase from the function in signalling.

\section{Short- and long-term repression}

We have shown previously that the establishment of the repressed state of catabolite-repressible genes is at least a two-step process consisting of a rapid, initial disappearance of the messengers and a long-term or sustained repression (de Winde et al., 1996). The new data confirm the (at least) two-stage nature of catabolite repression by adding a new mutant phenotype: switching of short-term repression into induction while at the same time long-term repression is still functional, at least partially. This phenotype is most pronounced in mutant $h \times k 1 \Delta b x k 2-129$ glk1 1 with glucose. Higher SUC2 mRNA levels $30 \mathrm{~min}$ after sugar addition were also observed in mutants 37 (with glucose), 39 (with glucose) and 129 (with fructose), in each case followed by diminished levels after $180 \mathrm{~min}$. Thus, different hexokinase mutations can affect either short- or longterm catabolite repression, or can affect either of the two more than the other. We suggest that short-term and long-term repression of catabolite-repressible genes are mediated by the sugar kinases or products produced by them, but using different signalling pathways.

\section{Glucose and fructose repression}

We have shown previously that repression by glucose or fructose has different requirements with respect to the sugar kinase isoform (de Winde et al., 1996). Mutant

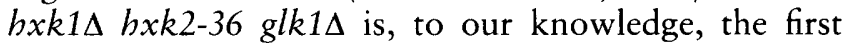
strain in which fructose repression of SUC2 is diminished while glucose repression is normal. This mutant is also more affected for fructose than for glucose phosphorylation and therefore this effect is in agreement with the previously established inverse correlation between sugar kinase and SUC2 repression. Still, this phenotype demonstrates that also mutations in $H X K 2$ can lead to diminished fructose repression.

\section{Role of sugar kinases in long-term catabolite repression}

The actual role of the sugar kinases in long-term catabolite repression has been a matter of controversy for many years. Our data challenge the presently accepted view that the role of the sugar kinases in catabolite repression is directly related to their catalytic activity. This view was based on the good inverse correlation between hexokinase and invertase activity in a collection of random $b x k 2$ mutants (Ma et al., 1989), $H X K 1-H X K 2$ fusions and $H X K 2$ promoter truncations (Rose et al., 1991). We do not see an inverse correlation between in vitro hexokinase and invertase activities in our mutants. We also do not see such an inverse correlation when we consider the rate of sugar phosphate accumulation as an indicator for in vivo hexokinase activity and when SUC2 expression was monitored more directly by Northern-blot analysis. Alleles 
$h x k 2-39$ and $h x k 2-129$ best demonstrate the lack of correlation. They behave similarly in terms of catabolite repression: short-term glucose induction is seen instead of repression but, at least to some extent, long-term repression and no apparent fructose repression are also seen. These two mutants have very different in vitro hexokinase activities, very different sugar phosphate accumulation profiles after sugar addition in vivo and different ethanol-production. rates. The lack of correlation between in vitro and apparent in vivo hexokinase activity and long-term repression also becomes apparent when the $h \times k 2-129$ allele is compared to $h \times k 2-$ 53. The $b x k 1 \Delta b x k 2-129$ glk1 $\Delta$ mutant has the highest fructose 6-phosphate level among all the mutants and, after a lag phase, a high ethanol-production rate on fructose but lacks fructose repression of SUC2. Mutant $h x k 1 \Delta h x k 2-53 \mathrm{glk} 1 \Delta$, on the other hand, clearly has lower initial fructose 6-phosphate levels and a similar ethanol-production rate when compared to $b x k 1 \Delta$ hxk2-129 glk1 , but does show normal fructose repression.

Since it has previously been difficult to separate the regulatory and catalytic functions of the sugar kinases, both appear to be closely related. One possible scenario could be related to substrate binding and recognition. What the mutant proteins Hxk2-39 and Hxk2-129 have in common (also partly with Hxk2-36), apart from their very similar defects in catabolite repression, is a dramatically altered proportion of the affinities for sugar and ATP, albeit in opposite directions. If binding of both substrates does not occur in the proper coordinated fashion, this might affect the nature or the timing of a conformational change in the protein. If such a conformational change were the trigger for (a) signalling pathway(s) some protein(s) should recognize this switch and this protein(s) would then remain to be identified. An alternative explanation is that the mutations identified in this study affect to different degrees the domain of the Hxk2 protein that would interact with a downstream component of the glucose-repression pathway.

\section{Role of sugar kinases in CAMP signalling}

We have shown previously that sugar phosphorylation, but not further metabolism of the sugar, is essential for: glucose- and fructose-induced activation of cAMP synthesis (Beullens et al., 1988). The sugar phosphate produced, however, did not seem to act as the trigger since no correlation was observed between the increase in glucose 6-phosphate and the increase in cAMP when cells were exposed to different glucose concentrations. In the present experiments there is a fair correlation between the increase in glucose 6-phosphate for the different hexokinase alleles, although this correlation is not perfect. For instance, the strains HXK1 GLK1 $h x k 2 \Delta$ and $h x k 1 \Delta b x k 2-37$ glk1 $1 \Delta$ have the same increase in glucose 6-phosphate but the former has a higher cAMP increase. Nevertheless, the data tend to indicate that the role of hexokinase in sugar-induced activation of cAMP synthesis is closely connected to its catalytic function. In that case, the conformational change in the protein during catalysis might trigger activation of a component of the cAMP pathway. As observed for the role of hexokinase in glucose repression, such a mechanism would tend to result in a general correlation between catalytic activity and regulatory activity for induction of cAMP synthesis. Only with mutations in very specific residues would this correlation disappear. Hence, as we have accomplished in this paper for hexokinase catalytic activity and glucose repression function, very specific selection schemes might be needed to find hexokinase mutants in which catalytic activity and cAMP-synthesis activation are separated. It has been suggested that the rapid glucose-induced mRNA disappearance involves cAMP-dependent protein kinase (Cereghino \& Scheffler, 1996). The data presented here appear to contradict this idea, since in our mutants there is no correlation between the cAMP spike and the ability to mediate short-term (or long-term) catabolite repression. The present results clearly demonstrate that glucose-repression activity and the capacity to activate the cAMP pathway are distinct properties of hexokinase.

\section{ACKNOWLEDGEMENTS}

We thank Willy Verheyden for excellent technical assistance. This work was supported by fellowships from the Fund for Scientific Research-Flanders (senior research assistant) to J.W., the Institute for Scientific and Technological Research (IWT) (post-doctoral research fellowship) to C.d.M., the Human Capital and Mobility Programme of the Commission of the European Union to J.H.d.W. (contract ERB CHBG CT93-0284) and by grants from the Fund for Scientific Research-Flanders, the Research Fund of the Katholieke Universiteit Leuven (concerted research actions) to J.M.T. and the Human Capital and Mobility Programme of the Commission of the European Union (contract ERB CHRX CT93-0265) to J.M.T. and J.R.

\section{REFERENCES}

Anderson, C. M., Stenkamp, R. E., McDonald, R. C. \& Steitz, T. A. (1978). A refined model of the sugar binding site of yeast hexokinase B. J Mol Biol 123, 207-219.

Bennett, W. S., Jr \& Steitz, T. A. (1980). Structure of a complex between yeast hexokinase A and glucose. II. Detailed comparisons of conformation and active site configuration with the native hexokinase B monomer and dimer. J Mol Biol 140, 211-230.

Beullens, M., Mbonyi, K., Geerts, L., Gladines, D., Detremerie, K., Jans, A. W. \& Thevelein, J. M. (1988). Studies on the mechanism of the glucose-induced cAMP signal in glycolysis and glucose repression mutants of the yeast Saccharomyces cerevisiae. Eur J Biochem 172, 227-231.

Bisson, L. F. \& Fraenkel, D. G. (1983). Involvement of kinases in glucose and fructose uptake in Saccharomyces cerevisiae. Proc Natl Acad Sci USA 80, 1730-1734.

Blázquez, M. A. \& Gancedo, C. (1995). Mode of action of the qcr 9 and cat 3 mutations in restoring the ability of Saccharomyces cerevisiae tps1 mutants to grow on glucose. Mol Gen Genet 249, 655-664. 
Blázquez, M. A., Lagunas, R., Gancedo, C. \& Gancedo, J. M. (1993). Trehalose 6-phosphate, a new regulator of yeast glycolysis that inhibits hexokinases. FEBS Lett 329, 51-54.

Bork, P., Sander, C. \& Valencia, A. (1992). An ATPase domain common to prokaryotic cell cycle proteins, sugar kinases, actin, and hsp 70 heat shock proteins. Proc Natl Acad Sci USA 89, $7290-7294$.

Cereghino, G. P. \& Scheffler, I. E. (1996). Genetic analysis of glucose regulation in Saccharomyces cerevisiae: control of transcription versus mRNA turnover. EMBO J 15, 363-374.

Crauwels, M., Donaton, M. C. V., Pernambuco, M. B., Winderickx, J., de Winde, J. H. \& Thevelein, J. M. (1997). The Sch9 protein kinase in the yeast Saccharomyces cerevisiae controls cAPK activity and is required for nitrogen activation of the fermentable-growth-medium-induced (FGM) pathway. Microbiology 143, 2627-2637.

Entian, K. D. (1980). Genetic and biochemical evidence for hexokinase PII as a key enzyme involved in carbon catabolite repression in yeast. Mol Gen Genet 178, 633-637.

Entian, K. D. \& Fröhlich, K. U. (1984). Saccharomyces cerevisiae mutants provide evidence of hexokinase PII as a bifunctional enzyme with catalytic and regulatory domains for triggering carbon catabolite repression. J Bacteriol 158, 29-35.

Entian, K. D., Kopetzki, E., Fröhlich, K. U. \& Mecke, D. (1984). Cloning of hexokinase isoenzyme PI from Saccharomyces cerevisiae: PI transformants confirm the unique role of hexokinase isoenzyme PII for glucose repression in yeasts. Mol Gen Genet 198, 50-54.

Ernandes, J. R., DeMeirsman, C., Rolland, F., Winderickx, J., DeWinde, J., Brandao, R. L. \& Thevelein, J. M. (1998). During the initiation of fermentation overexpression of hexokinase PII in yeast transiently causes a similar deregulation of glycolysis as deletion of TPS1. Yeast 14, 255-269.

Gancedo, J. M. (1998). Yeast carbon catabolite repression. Microbiol Mol Biol Rev 62, 334-361.

Gancedo, J. M. \& Gancedo, C. (1997). Gluconeogenesis and catabolite inactivation. In Yeast Sugar Metabolism: Biochemistry, Genetics, Biotechnology and Application, pp. 359-377. Edited by F. K. Zimmermann \& K. D. Entian. Lancaster: Technomic:

Gidh-Jain, M., Takeda, J., Xu, L. Z. \& 17 other authors (1993). Glucokinase mutations associated with non-insulin-dependent (type 2) diabetes mellitus have decreased enzymatic activity: implications for structure/function relationships. Proc Natl Acad Sci USA 90, 1932-1936.

Goldstein, A. \& Lampen, J. O. (1975). $\beta$-D-fructofuranoside hydrolase from yeast. Methods Enzymol 42, 504-511.

Hardie, D. G., Carling, D. \& Carlson, M. (1998). The AMPactivated/SNF1 protein kinase subfamily: metabolic sensors of the eukaryotic cell? Annu Rev Biochem 67, 821-855.

Heidrich, K., Otto, A., Behlke, J., Rush, J., Wenzel, K. W. \& Kriegel, T. (1997). Autophosphorylation-inactivation site of hexokinase 2 in Saccharomyces cerevisiae. Biochemistry 36, 1960-1964.

Herrero, P., Galindez, J., Ruiz, N., Martinez Campa, C. \& Moreno, F. (1995). Transcriptional regulation of the Saccharomyces cerevisiae HXK1, HXK2 and GLK1 genes. Yeast 11, 137-144.

Hohmann, S. (1987). Physiologische und molekulargenetische Untersuchungen zur Saccharosevergärung der Hefe Saccharomyces cerevisiae. $\mathrm{PhD}$ thesis, Technische Hochschule Darmstadt. Hohmann, S., Neves, M. J., de Koning, W., Alijo, R., Ramos, J. \& Thevelein, J. M. (1993). The growth and signalling defects of the ggs1 ( $f d p 1 /$ byp1) deletion mutant on glucose are suppressed by a deletion of the gene encoding hexokinase PII. Curr Genet 23, 281-289.

Hohmann, S., Bell, W., Neves, M. J., Valckx, D. \& Thevelein, J. M. (1996). Evidence for trehalose 6-phosphate-dependent and -independent mechanisms in the control of sugar influx into yeast glycolysis. Mol Microbiol 20, 981-991.

de Koning, W. \& van Dam, K. (1992). A method for the determination of changes of glycolytic metabolites in yeast on a subsecond time scale using extraction at neutral $\mathrm{pH}$. Anal Biochem 204, 118-123.

Laemmli, U. K. (1970). Cleavage of structural proteins during the assembly of the head of bacteriophage T4. Nature 277, 680-685.

Lobo, Z. \& Maitra, P. K. (1977). Genetics of yeast hexokinase. Genetics 86, 727-744.

Luyten, K., de Koning, W., Tesseur, I., Ruiz, M. C., Ramos, J., Cobbaert, P., Thevelein, J. M. \& Hohmann, S. (1993). Disruption of the Kluyveromyces GGS1 gene causes inability to grow on glucose and fructose and is suppressed by mutations that reduce sugar uptake. Eur J Biochem 217, 701-713.

Ma, H., Bloom, L. M., Walsh, C. T. \& Botstein, D. (1989). The residual enzymatic phosphorylation activity of hexokinase PII mutants is correlated with glucose repression in Saccharomyces cerevisiae. Mol Cell Biol 9, 5643-5649.

Maitra, P. K. \& Lobo, Z. (1983). Genetics of yeast glucokinase. Genetics 105, 501-515.

Ronne, H. (1995). Glucose repression in fungi. Trends Genet 11, $12-17$.

Rose, M., Albig, W. \& Entian, K. D. (1991). Glucose repression in Saccharomyces cerevisiae is directly associated with hexose phosphorylation by hexokinase PI and PII. Eur J Biochem 199, 511-518.

Schirch, D. M. \& Wilson, J. E. (1987). Rat brain hexokinase: amino acid sequence at the substrate hexose binding site is homologous to that of yeast hexokinase. Arch Biochem Biophys 254, 385-396.

Sherman, F., Fink, G. R. \& Hicks, J. B. (1983). Methods in Yeast Genetics. Cold Spring Harbor, NY: Cold Spring Harbor Laboratory.

Sierkstra, L. N., Nowen, N. P., Verbakel, J. M. A. \& Verrips, C. T. (1992). Analysis of glucose repression in Saccharomyces cerevisiae by pulsing glucose to galactose limited continuous culture. Yeast 8, 1077-1087.

Smits, H. P., Smits, G. J., Postma, P. W., Walsh, M. C. \& van Dam, K. (1996). High-affinity glucose uptake in Saccharomyces cerevisiae is not dependent on the presence of glucosephosphorylating enzymes. Yeast 12, 439-447.

Thevelein, J. M. \& Beullens, M. (1985). Cyclic AMP and the stimulation of trehalose activity in the yeast Saccharomyces cerevisiae by carbon source, nitrogen source and inhibitors of protein synthesis. J Gen Microbiol 131, 3199-3209.

Thevelein, J. M. \& Hohmann, S. (1995). Trehalose synthase, guard to the gate of glycolysis in yeast? Trends Biochem Sci 20, 3-10.

Thevelein, J. M., Beullens, M., Honshoven, F., Hoebeeck, G., Detremerie, K., den Hollander, J. A. \& Jans, A. W. H. (1987). Regulation of the cAMP level in the yeast Saccharomyces cerevisiae: intracellular $\mathrm{pH}$ and the effect of membrane depolarizing compounds. J Gen Microbiol 133, 2191-2196.

Thomas, B. J. \& Rothstein, R. J. (1989). Elevated recombination rates in transcriptionally active DNA. Cell 56, 619-630.

Towbin, H., Staehelin, T. \& Gordon, J. (1979). Electrophoretic 
transfer of proteins from polyacrylamide gels to nitrocellulose sheets: procedure and some applications. Proc Natl Acad Sci USA 79, 4350-4354.

Van Aelst, L., Hohmann, S., Bulaya, B. \& 15 other authors (1993). Molecular cloning of a gene involved in glucose sensing. $\mathrm{Mol}$ Microbiol 8, 927-943.

de Winde, J. H., Crauwels, M., Hohmann, S., Thevelein, J. M. \& Winderickx, J. (1996). Differential requirement of the yeast sugar kinases for sugar sensing in the establishing the cataboliterepressed state. Eur J Biochem 241, 633-643.
Xu, L. Z., Harrison, R. W., Weber, I. T. \& Pilkis, S. J. (1995). Human $\beta$-cell glucokinase: dual role of Ser-151 in catalysis and hexose affinity. J Biol Chem 270, 9939-9946.

Zimmermann, F. K. \& Entian, K. D. (1997). Yeast Sugar Metabolism: Biochemistry, Genetics, Biotechnology and Applications. Lancaster, PA: Technomic.

Received 3 August 1998; revised 5 November 1998; accepted 16 November 1998. 